K Sundaram et al.

\title{
IкB $\zeta$ is constitutively expressed in human and murine airway epithelium
}

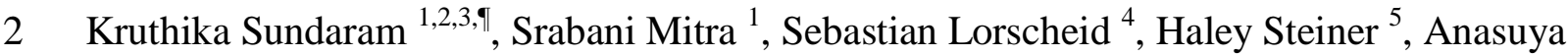

3 Sarkar $^{1}$, Konstantin Shilo ${ }^{6}$, Prosper N. Boyaka ${ }^{5}$, Klaus Schulze-Osthoff ${ }^{4,7}$, and Mark D.

4 Wewers $^{1}$

1 Pulmonary, Allergy, Critical Care and Sleep Medicine, Davis Heart and Lung Research Institute, Department of Internal Medicine, Ohio State University Medical Center, Columbus, Ohio, USA.

2 King's College London, King's Health Partners, King's College Hospital NHS Foundation

11 Trust, School of medicine, Division of Asthma, Allergy and Lung Biology, Department of

12 Immunological Medicine, Denmark Hill, London SE5 9RS, UK

3 Viapath, King's College Hospital, Denmark Hill, London SE5 9RS, UK

4 The Interfaculty Institute of Biochemistry, Department of Molecular Medicine, University of

Tübingen, Tübingen, Germany.

5 Department of Veterinary Biosciences, The Ohio State University, Columbus, Ohio, USA.

6 Department of Pathology, The Ohio State University College of Medicine, Columbus, Ohio, USA.

7 The German Cancer Consortium (DKTK) and German Cancer Research Center, Heidelberg, Germany.

${ }^{\text {II }}$ Corresponding Author Kruthika Sundaram PhD Scientific Research Officer Viapath

First floor, Bessemer Wing, King's College Hospital Denmark Hill, London SE5 9RS United Kingdom Phone: +4420 32999000 Ext 38756 Email:sundaram.25@osu.edu 
K Sundaram et al.

\section{ABSTRACT}

44 AIM: $\mathrm{I} \kappa \mathrm{B} \zeta$ is a transcriptional factor induced in immune cells upon Toll-like receptor (TLR)

45 activation. Recent studies demonstrate unconventional, constitutive expression of $\mathrm{I} \kappa \mathrm{B} \zeta$ in

46 epithelium of mouse skin and eyes, possibly reflecting continuous activation of TLRs by

47 pathogen-associated molecular patterns (PAMPs). In this context, the lung epithelium which

48 constitutes another important barrier also expresses $\mathrm{I} \kappa \mathrm{B} \zeta$ but may not be as actively exposed to

49 pathogens as skin and eyes. Our aim was to determine if $\mathrm{I} \kappa \mathrm{B} \zeta$ expression in the lungs is

50 constitutive or induced. SIGNIFICANCE: $\mathrm{I} \kappa \mathrm{B} \zeta$ is linked to lung disorders due to its role in

51 regulating protective cytokines and antimicrobial peptides in airway epithelium and can therefore

52 be a potential biomarker and a key therapeutic target. METHODS: We evaluated IкB $\zeta$

53 expression in airway epithelia of healthy humans and three kinds of mice: normal, gnotobiotic

54 and $\mathrm{Nfkbiz}^{-/}$knockout, using immunostaining and immunoblotting. RESULTS:

55 Immunohistochemistry of ciliated airway epithelial cells in healthy humans and normal mice was

56 positive for $\mathrm{I} \kappa \mathrm{B} \zeta$. The pathogen free airway cells from gnotobiotic mice also stained positive,

57 suggesting that lung epithelial $\mathrm{I} \kappa \mathrm{B} \zeta$ expression does not require induction by PAMPs. Although

58 lung epithelia from $\mathrm{Nfkbiz}^{-/}$knockout mice also stained positive, this knockout may not have

59 eliminated exons 3-4 and 9-14, and so did not provide the specificity control for the IкB $\zeta$

60 antiserum. Importantly, immunoblotting tissue homogenates from gnotobiotic mouse lungs and

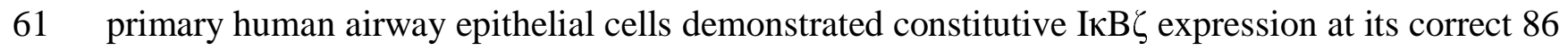

$62 \mathrm{kDa}$ size.

63 CONCLUSIONS: Our data demonstrates constitutive expression of $\mathrm{I} \kappa \mathrm{B} \zeta$ protein in airway

64 epithelium, indicating a potential role for this molecule in lung homeostasis.

65 Keywords: NFKBIZ, IкB $\zeta$, airway epithelium, constitutive expression. 
K Sundaram et al.

\section{BACKGROUND}

$\mathrm{I} \kappa \mathrm{B} \zeta$, also known as MAIL (molecule with ankyrin repeats induced by LPS) or INAP (IL-1-inducible nuclear ankyrin-repeat protein), is a protein encoded by the gene NFKBIZ (1-3). It predominantly functions as a transcription factor to regulate the expression of many

71 downstream molecules that are involved in host defense, such as IL-6, IL-12 (p40), granulocyte-

72 macrophage colony-stimulating factor, neutrophil gelatinase-associated lipocalin, human $\beta$

73 defensin 2 and monocyte chemoattractant protein 1 (4-9). I $\kappa \mathrm{B} \zeta$ is a homolog of the I $\kappa \mathrm{B}$ group of

74 proteins, due to the presence of an ankyrin repeat region in its carboxy-terminus, with the help of

75 which it binds to NFkB p50 homodimers, typically inside the nucleus of the cell $(1,3)$. The

76 amino-terminus of $\mathrm{I} \kappa \mathrm{B} \zeta$ encodes a transcriptional activation domain and a nuclear localization

77 sequence. There are two known isoforms of the protein: long ( $\mathrm{I} \kappa \mathrm{B} \zeta-\mathrm{L})$ and short (I $\mathrm{B} \zeta-\mathrm{S})$, of

78 which $\mathrm{I} \kappa \mathrm{B} \zeta-\mathrm{L}$ is predominantly expressed at the protein level (1). Apart from its role as a

79 transcription factor, $\mathrm{I} \kappa \mathrm{B} \zeta$ is known to exhibit other functions. For instance, I $\mathrm{I} \mathrm{B} \zeta$ modulates

80 chromatin remodeling, by selectively regulating $\mathrm{H} 3 \mathrm{~K} 4$ trimethylation and the assembly of the

81 pre-initiation complex at the promoter of downstream genes (10).

82 A majority of studies have described $\mathrm{I} \kappa \mathrm{B} \zeta$ as a primary, early response gene induced rapidly

83 following TLR stimulation of immune cells including mononuclear phagocytes, NK cells, T and

84 B lymphocytes $(6,8,11)$. Nevertheless, constitutive expression of the NFKBIZ gene has been

85 demonstrated in mucosal and epidermal sites that interact with the outside environment $(12,13)$,

86 indicating the possibility of chronic exogenous challenges inducing stable $\mathrm{I} \kappa \mathrm{B} \zeta$ expression in

87 these cells. In support of this concept, $\mathrm{I} \kappa \mathrm{B} \zeta$ knockout mice display severe inflammation of the 88 epithelium lining their eyes and skin (13-15), suggesting the importance of $\mathrm{I} \kappa \mathrm{B} \zeta$ in maintaining

89 immune homeostasis of the host. Interestingly, IкB $\zeta$ has been demonstrated to regulate the 90 expression of pro-inflammatory cytokines and anti-microbial peptides in airway epithelial cells 
K Sundaram et al.

91 that line the respiratory mucosal surface, in mammalian models of asthma and pneumonia $(4,5$,

92 16). Studies have shown baseline $N F K B I Z$ gene expression in lung tissues $(3,14)$, although the

93 induction of $\mathrm{I} \kappa \mathrm{B} \zeta$ protein expression in the airway epithelium in response to environmental

94 agonists remains understudied. The present study therefore explored the expression of I $\kappa \mathrm{B} \zeta$ in

95 both human and mouse airway epithelial cells. Using immunohistochemistry (IHC), we detected

96 positive staining in the nuclei of ciliated epithelial cells in the upper airway tract of human and

97 wildtype gnotobiotic mice. Immunoblotting of tissue homogenates from gnotobiotic mice and

98 primary human airway epithelial cells demonstrated constitutive expression of $\mathrm{I} \kappa \mathrm{B} \zeta$. Thus, our

99 results support a role for $\mathrm{I} \kappa \mathrm{B} \zeta$ in airway protection.

100

101

102

103

104

105

106

107

108

109

110

111

112

113 
K Sundaram et al.

\section{MATERIALS AND METHODS}

\section{Reagents and antibodies}

116 The reagents were obtained from the following sources: IL-1 receptor antagonist, IL-1Ra

117 (Amgen), penicillin-streptomycin (Invitrogen), fetal bovine serum FBS (Atlanta Biologicals),

118 Dulbecco modified Eagle medium low glucose DMEM (Invitrogen), Bronchial Epithelial

119 Growth Medium BEGM bullet kit (Lonza), Bronchial Air Liquid Interface B-ALI media

120 (Lonza), Small airway epithelial cell media and detach kit (PromoCell), recombinant human IL-

$1211 \beta$ was purchased from $\mathrm{R} \& \mathrm{D}$ systems. Rabbit antiserum against $\mathrm{I} \kappa \mathrm{B} \zeta$ was generated in our

122 laboratory using recombinant protein expressed in Escherichia coli (8) (17). Commercial

123 antibodies against I $\mathrm{I} B \zeta$ were purchased from Thermo Fisher Scientific (PA5-52703), Sigma

124 Aldrich (HPA010547), Abcam (ab221914) and LS Biosciences (LS-C294627). Scrambled

125 siRNA control and $\operatorname{siI} \kappa \mathrm{B} \zeta$ (sequence UGAUGGACCUGCUUGCAAA) were purchased from

126 Dharmacon Thermo Scientific. Lamin B1 antibody and beta-actin antibody (monoclonal clone

127 C4) were purchased from Santa Cruz Biotechnology and MP Biomedicals respectively.

\section{Mouse lung tissue}

129 Lungs were harvested from wildtype B10RIII mice weighing 17-20 grams and lungs and

130 tracheae were isolated from 9-12 weeks old gnotobiotic male Swiss Webster mice, weighing 17-

13121 grams. Wildtype and $\mathrm{Nfkbiz}^{-/-}$knockout mice were allowed free access to food and water,

132 while the gnotobiotics were bred and maintained in sterile isolators. All animal experiments were

133 performed according to animal protocols approved by the Animal Care Use Committee of the

134 Ohio State University College of Medicine. Lungs from wildtype mice were equivalently

135 inflated with an intratracheal injection of a similar volume of $4 \%$ paraformaldehyde solution

136 (Sigma) to preserve the pulmonary architecture. The lungs were then embedded in paraffin. The 
K Sundaram et al.

137 sections of all organs collected were then stained with hematoxylin and eosin or with anti-IкB $\zeta$

138 antiserum, along with appropriate controls.

139 Human lung tissue

140 Anonymized human lung tissue slides were provided by the Ohio State University

141 Department of Pathology, Tissue Archive Service. Other investigators may have received

142 specimens from the same subjects.

\section{Immunohistochemistry}

144 The lung tissue slides were labeled with $\mathrm{I} \kappa \mathrm{B} \zeta$ antiserum at the specified dilution with 145 anti-rabbit secondary antibody at a 1:1000 dilution at the Comparative Pathology and Mouse 146 Phenotyping Shared Resource, at the Veterinary school of OSU.

\section{Cell culture}

BEAS2B and HeLa cells (purchased from ATCC) were maintained in DMEM low

149 glucose, supplemented with $10 \%$ FBS and $1 \%$ penicillin-streptomycin, in a $37^{\circ} \mathrm{C}$ humidified

150 incubator with $5 \% \mathrm{CO}_{2}$. Monocytes were obtained from fresh buffy coats purchased from the

151 American Red Cross. Monocytes were purified from blood by Histopaque density gradient

152 centrifugation using lymphocyte separation media (Cellgro) followed by CD14 positive selection

153 as described previously (8). Purified monocytes and monocytic cell line THP-1 were both

154 maintained in RPMI1640 supplemented with 10\% FBS. Primary human bronchial epithelial cells

155 (HBECs), purchased from Lonza were allowed to differentiate in B-ALI media in 24-well inserts

$156(6.5 \mathrm{~mm})$ as per manufacturer's instructions. HBECs $\left(5 \times 10^{4}\right.$ cells $\left./ 33 \mathrm{~mm}^{2}\right)$ were stimulated with

157 rhIL-1 $\beta(10 \mathrm{ng} / \mathrm{ml})$ for 3 hours, with or without 30 minutes of pretreatment with IL-1Ra

158 (10ug/ml). Cells are then lysed and immunoblotted. 
K Sundaram et al.

160

161

162

163

164

165

166

167

168

169

170

171

172

173

174

175

176

177 blocking. Our laboratory made anti-IкB $\zeta$ antiserum was incubated with 1000-2000 times excess

178 by volume of the THP-1 NE at room temperature for 1-2 hours. This antigen-antibody mixture

179 was then used for immunoblotting or immunostaining.

\section{Immunostaining of BEAS2B cells}

\section{Generation of recombinant IKB $\zeta$}

I $\kappa \mathrm{B} \zeta-\mathrm{S}$ was cloned in pET32a bacterial vector with an $\mathrm{N}$-terminal thioredoxin tag and a C-terminal histidine tag and then over expressed in BL21DE3RIL strain of bacteria. The over expressed protein was purified using HisPur cobalt resin and dialyzed into a $20 \mathrm{mM}$ Tris buffer, pH 7.5 with $150 \mathrm{mM} \mathrm{NaCl}$ and $1 \mathrm{mM}$ beta mercaptoethanol, using a Slide-a-lyzer, both from Thermo Scientific. Expression of purified $\mathrm{rI} \mathrm{BB} \zeta-\mathrm{S}$ was confirmed using immunoblotting with both anti-IкB $\zeta$ antiserum and His antibody.

\section{Blocking non-specificity of anti-IKB $\zeta$ antiserum using THP-1 extracts}

Unstimulated THP-1 cells ( $\sim 7 \times 10^{7}$ cells) that do not express IкB $\zeta$ were washed to remove culture media and re-suspended in $10 \mathrm{ml}$ of homogenization buffer $(0.25 \mathrm{M}$ sucrose, $1 \mathrm{mM}$ EDTA, 20mM HEPES pH 7.5) with protease inhibitors mixture (Sigma-Aldrich). The cells were then dounced on ice and centrifuged at low speed ( 100rpm) to remove unbroken cells. The supernatant was centrifuged at high speed ( 2000rpm) and was separated into the cellular extract (CE) fraction and the intact nuclei. The intact nuclei that pellet out were then re-suspended in $1 \mathrm{ml}$ of Buffer W (20mM HEPES pH 7.5, 10mM KCl, $1.5 \mathrm{mM} \mathrm{MgCl}_{2}, 1 \mathrm{mM}$ EGTA, $1 \mathrm{mM}$ EDTA) with protease inhibitors, sonicated on ice for 10-15 minutes and centrifuged at high speed to yield the nuclear pellet $(\mathrm{Nu})$ and the nuclear extract $(\mathrm{NE})$ that was then used for

BEAS2B cells $\left(10^{5}\right.$ cells $\left./ \mathrm{ml}\right)$ were seeded onto sterile coverslips placed inside 12 well plates and cultured overnight. The cells were then stimulated with IL-1 $\beta(10 \mathrm{ng} / \mathrm{ml})$ for 3-4 hours. 
K Sundaram et al.

183 The media was removed and ice cold $100 \%$ methanol was slowly added to the coverslips to fix

184 the cells. The cells were left in methanol at $4^{\circ} \mathrm{C}$ overnight. The cells were then immunostained

185 using the Vectastain Elite ABC kit from Vector laboratories, as per manufacturer's instructions.

186 The primary antibody used was either anti-IкB $\zeta$ antiserum alone at 1:2000 dilution or with anti-

$187 \mathrm{I} \kappa \mathrm{B} \zeta$ antiserum incubated with excess THP-1 NE.

\section{Immunoprecipitation}

Human monocytes were plated in $10 \mathrm{ml}$ petri dishes and were stimulated with $1 \mu \mathrm{g} / \mathrm{ml}$

LPS for 3 h. The cells were then lysed in RIPA buffer (50mM Tris [pH 7.5], 1\% sodium

191 deoxycholate, $1 \mathrm{mM}$ EDTA, $1 \mathrm{mM} \mathrm{NaF}, 150 \mathrm{mM} \mathrm{NaCl}, 1 \% \mathrm{NP}-40$ ) with the protease inhibitor

192 cocktail mix, $10 \mathrm{mM}$ methoxysuccinyl-Ala-Ala-Pro-Val-chloromethyl ketone, $1 \mathrm{mM}$

193 phenylmethylsulfonyl fluoride (Sigma-Aldrich), and $3 \mathrm{mM}$ sodium orthovanadate. Cell lysates

194 were pre-cleared using protein A beads (Invitrogen). Pre-cleared lysates were

195 immunoprecipitated using anti-lamin B1 antibody and these immunoprecipitated samples were

196 incubated with protein A-sepharose beads (Invitrogen) for $2 \mathrm{~h}$ at $4{ }^{\circ} \mathrm{C}$, followed by centrifugation

197 at $16,000 \times \mathrm{g}$ for $5 \mathrm{~min}$ and washing. The proteins were eluted with $2 \mathrm{X}$ SDS-PAGE sample

198 buffer at $95^{\circ} \mathrm{C}$ for $10 \mathrm{~min}$ and were separated by SDS-PAGE and blotted with anti-lamin B1

199 antibody and anti- I $\kappa \mathrm{B} \zeta$ antiserum.

$200 \quad 2 D$ gel analysis

201 IL-1 $\beta$ stimulated HeLa cells were immunoprecipitated with anti-IKB $\zeta$ antiserum as

202 described above. The immunoprecipitated samples were run on a 2D gel electrophoresis system

203 at the OSU's Campus Chemical Instrument Center (CCIC) mass spectrometry and proteomics

204 core facility, on an 18-cm 4-7 IPG strip, since the predicted isoelectric point for I $\mathrm{B} \zeta$ was around

205 6. The separated proteins were then either transferred onto a PVDF membrane and 
K Sundaram et al.

206 immunoblotted with anti-I $\mathrm{kB} \zeta$ antiserum or were further processed for mass spectrometry

207 analysis.

\section{Mass spectrometry}

209 The 2D gel was stained with Coomasie blue and bands corresponding to $86 \mathrm{kDa}$ (the size

210 of $\mathrm{I} \kappa \mathrm{B} \zeta-\mathrm{L})$ and $68 \mathrm{kDa}$ (the size of $\mathrm{I} \kappa \mathrm{B} \zeta-\mathrm{S}$ ) were excised and submitted to OSU's proteomics

211 facility. Samples underwent automated in-gel protein digestion in the MassPrep station. Briefly,

212 they were destained, reduced and incubated with iodoacetamide in ammonium bicarbonate for 20

213 min at $37{ }^{\circ} \mathrm{C}$. They were then washed with ammonium bicarbonate/water and dehydrated with

214 acetonitrile. The extracted proteins were in-gel digested with $6 \mathrm{ng} / \mu \mathrm{l}$ trypsin in $50 \mathrm{mM}$

215 ammonium bicarbonate for $5 \mathrm{~h}$ at $37{ }^{\circ} \mathrm{C}$. Mass spectra of resulting peptides were recorded on the

216 ESI-TRAP spectrometer. The resulting peptides were matched with their corresponding proteins

217 with ProFound by searching the non-redundant data base maintained at the NCBI

218 (www.ncbi.nlm.nih.gov). The cut off for ion score was kept at 20, where the score was

219 calculated as $-10 * \log (\mathrm{P})$, and $\mathrm{P}$, the probability that the observed match is a random event. The

220 following parameters were used for the search at Pro-Found: taxa Homo sapiens, fixed

221 modifications carbamidomethyl, variable modifications oxidation, allowed incomplete cleavages

222 2, monoisotopic masses, and a peptide mass tolerance of $\pm 1.8 \mathrm{Da}$.

\section{Tissue homogenization}

224 Tissue chunks of lungs or trachea from mice were added to SDS lysis buffer (100 mM

$225 \mathrm{NaCl} 500 \mathrm{mM}$ Tris, $\mathrm{pH} 8.0$ 10\% SDS) and vortexed for about 30 minutes till the chunks

226 dissolved. The tissues were then syringed approximately 20 times and boiled at $100^{\circ} \mathrm{C}$ for

227 complete extraction. The tissue extracts were then protein estimated and immunoblotted for I $\mathrm{kB} \zeta$

228 expression. 
K Sundaram et al.

Small interfering RNA (siRNA) mediated knockdown of IKB $\zeta$

BEAS2B cells were transfected with 100 pmol of scrambled siRNA control or siRNA

231 specific to I $\kappa \mathrm{B} \zeta$ using lipofectamine (Invitrogen). After 20 hours, media was replaced, following

232 which the cells were stimulated with rIL-1 $\beta(10 \mathrm{ng} / \mathrm{ml})$ for 4 hours.

\section{Preparation of cell lysates and immunoblotting}

Cells were lysed in TN1 lysis buffer (50 mM Tris-HCl, pH 8.0, $125 \mathrm{mM} \mathrm{NaCl,} 10 \mathrm{mM}$ ethylene diaminetetraacetic acid (EDTA), $10 \mathrm{mM} \mathrm{NaF,} 10 \mathrm{mM}$ sodium pyrophosphate, $1 \%$ Triton $\mathrm{X}-100)$ with the protease and phosphatase inhibitor cocktails. The extracts were incubated on ice for 15 minutes, syringed $10-15$ times and centrifuged at $16,000 \mathrm{~g}$ for 10 minutes. The lysates were transferred to a new Eppendorf tube and total protein in each sample was determined using Lowry assay (Bio-Rad). Equal protein (20ug per lane) was loaded onto a NuPAGE 7\% Trisacetate gel (Invitrogen). The separated proteins were transferred onto polyvinylidene difluoride membranes. The membranes were blocked with $10 \%$ nonfat milk, incubated overnight at $4{ }^{\circ} \mathrm{C}$ with the primary antibody, washed and then stained with appropriate peroxidase-conjugated secondary antibody for an hour. The protein bands were visualized by enhanced chemiluminescence substrate (GE Healthcare) by autoradiography. 
K Sundaram et al.

\section{RESULTS}

\section{Anti-IkB $\zeta$ antiserum detects $I k B \zeta$}

255 The first step to address the aim of the study was to find an antibody that would detect

$256 \mathrm{I} \kappa \mathrm{B} \zeta$ with high sensitivity and specificity. We started by validating the anti-I $\kappa \mathrm{B} \zeta$ antiserum

257 generated in our laboratory. To do this, we transfected BEAS2B cells with siRNA specific to

$258 \mathrm{I} \mathrm{I} \mathrm{B} \zeta$ and stimulated them with rIL-1 $\beta$ for 4 hours. The anti-I $\kappa \mathrm{B} \zeta$ antiserum detected the expected

259 pattern of $\mathrm{I} \kappa \mathrm{B} \zeta-\mathrm{L}$ expression at the correct size of $86 \mathrm{kDa}$. The protein was induced in response

260 to IL-1 $\beta$ stimulation which was blocked in the presence of si-I $\mathrm{B} \zeta$ (Figure S1A). However, the

261 antiserum also detected non-specific proteins. We then tested a panel of commercial antibodies

262 from different manufacturers. None of them detected I $\kappa$ B $\zeta$ with the same sensitivity as our

263 laboratory generated anti-IкB $\zeta$ antiserum (Figure S1B). Additionally, they also detected non-

264 specific bands. We hence used our anti-IкB $\zeta$ antiserum for all further experiments in this study.

\section{$265 \quad$ IkB $\zeta$ staining observed in mammalian lung tissue}

266 To understand $\mathrm{I} \kappa \mathrm{B} \zeta$ 's behavior in airway epithelial cells, we immunostained lung tissue

267 slices from normal human subjects (Figure 1A) and from wild type mice (Figure 1B) with the 268 anti-I $\kappa \mathrm{B} \zeta$ antiserum. We observed a significant increase in staining with anti-I $\mathrm{B} \zeta$ antiserum

269 compared to the preimmune control serum and the secondary antibody negative control. This

270 signal was predominantly present in the nuclei of ciliated epithelial cells lining the central

271 airways, which was the expected cellular location for $\mathrm{I} \kappa \mathrm{B} \zeta$ due to its known role in transcription

272 regulation.

273 Gnotobiotic lungs stain positive for IkB $\zeta$ expression 
K Sundaram et al.

Because I $\mathrm{I} B \zeta$ is considered to be a classic example of an early response gene, we asked if

275 the baseline $\mathrm{I} \kappa \mathrm{B} \zeta$ expression was due to exposure to bacterial PAMPs. Therefore, we examined

276 lung tissue from gnotobiotic (germ-free) mice. Surprisingly, the ciliated epithelial cells of the

277 gnotobiotics also immunostained for $\mathrm{I} \kappa \mathrm{B} \zeta$ expression, both in the major bronchi (Figure 2A) and

278 trachea (Figure S2), suggesting that expression of $\mathrm{I} \kappa \mathrm{B} \zeta$ in the airway epithelium does not require

279 induction by bacterial PAMPs.

280 Anti-IkB $\zeta$ antiserum also detects lamin B1

281 To further validate these results, we immunostained lung tissue samples from $\mathrm{Nfkbiz}^{-/-}$

282 knockout mice (Figure 2B). Unexpectedly, we obtained a positive signal. However, this

283 knockout (13) may not have eliminated exons 3-4 and 9-14, and so did not provide the

284 specificity control for the IאB $\zeta$ antiserum.

In an attempt to prove specificity of the antiserum, we used column-purified recombinant

$286 \mathrm{I} \kappa \mathrm{B} \zeta-\mathrm{S}$ expressed in bacterial cells (Figure 3A) to block $\mathrm{I} \kappa \mathrm{B} \zeta$ detection by anti-I $\kappa \mathrm{B} \zeta$ antiserum.

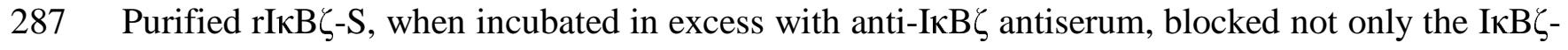

288 L 86kDa band but also all of the nonspecific bands (Figure 3B), as observed previously in our

289 group (17). This result therefore still required further specificity confirmation.

290 To evaluate what other proteins were detected by the anti-I $\mathrm{B} \zeta$ antiserum, we performed

$2912 \mathrm{D}$ electrophoresis on samples obtained by immunoprecipitation using the anti-I $\kappa \mathrm{B} \zeta$ antiserum

292 from lysates of HeLa cells stimulated with IL-1 $\beta$ (Figure 4A). Mass spectrometry analysis of the

293 cluster of spots identified $\mathrm{I} \kappa \mathrm{B} \zeta-\mathrm{L}$ at the expected size of $86 \mathrm{kDa}$ towards the acidic end of the $\mathrm{pH}$

294 strip. Interestingly, spots that ran at $68 \mathrm{kDa}$ with isoelectric points close to neutral $\mathrm{pH}$ were

295 identified as the nuclear protein lamin $\mathrm{B} 1$ and not $\mathrm{I} \kappa \mathrm{B} \zeta-\mathrm{S}$ which also runs at $68 \mathrm{kDa}$. The cross

296 reactivity with lamin B1 was further confirmed when lamin B1 was detected by anti-IкB $\zeta$ 
K Sundaram et al.

antiserum in the sample immunoprecipitated with anti-lamin B1 antibody from lysates of monocytes stimulated with LPS (Figure 4B).

Since our attempts to specifically block $\mathrm{I} \kappa \mathrm{B} \zeta$ detection alone by preblocking our anti-

$300 \mathrm{I} \kappa \mathrm{B} \zeta$ antiserum with recombinant $\mathrm{I} \kappa \mathrm{B} \zeta$ failed, we tried to remove all the non-specific $\operatorname{IgG}$

301 molecules in our anti-I $\kappa \mathrm{B} \zeta$ antiserum, leaving the $\mathrm{IgGs}$ specific to $\mathrm{I} \kappa \mathrm{B} \zeta$ available for binding. To

302 do this, we incubated anti-IkB $\zeta$ antiserum with an excess of nuclear extracts (NE) from

303 unstimulated THP-1 cells that contained lamin B1 but no I $\kappa$ B $\zeta$, i.e., the non-specific antigens

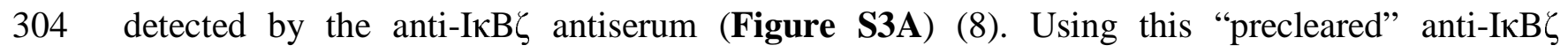

305 antiserum on IL-1 $\beta$ activated BEAS2B cells significantly blocked the immunoblot and

306 immunostaining for lamin B1 (Figure S3B \& C) without reducing the $\mathrm{I} \kappa \mathrm{B} \zeta$ signal. However,

307 THP-1 extract did not completely block the non-specific IgGs in the anti-IкB $\zeta$ antiserum, despite

308 its presence in huge excess, as seen in the western blot and the immunostain (Figure S3B \& top

309 panel of S3C).

310 Immunoblotting demonstrates definitive constitutive IkB $\zeta$ expression in lung epithelium

311 We then resorted to immunoblotting with anti-I $\kappa \mathrm{B} \zeta$ antiserum to perform size-based

312 detection of specific I $\kappa \mathrm{B} \zeta$ expression and thus definitely demonstrate its constitutive expression

313 in lung epithelium. Tissue homogenates from gnotobiotic mice showed $\mathrm{I} \kappa \mathrm{B} \zeta$ - $\mathrm{L}$ expression at the

314 correct molecular size $(\sim 86 \mathrm{kDa})$ both in the lungs and the trachea (Figure 5A). We then

315 compared the expression of the protein in differentiated primary human bronchial epithelial cells

316 (HBEC). HBECs displayed baseline I $\mathrm{KB} \zeta$ expression in the untreated controls (Figure 5B) that

317 was further induced by IL-1 $\beta$ and this induction was inhibited in the presence of IL-1 receptor

318 antagonist (IL-1Ra). 
K Sundaram et al.

\section{DISCUSSION}

324 Various groups have explored the role of $\mathrm{I} \kappa \mathrm{B} \zeta$ in regulating respiratory immunity. I $\mathrm{I} \mathrm{B} \zeta$

325 has been demonstrated to modulate the expression of pro-inflammatory cytokines like GMCSF

326 and IL-6 and anti-microbial peptides like NGAL in airway epithelial cells that line the

327 respiratory mucosal surface $(4,5,7,16)$. This is relevant to lung defense because GMCSF is

328 critical for the maintenance of lung surfactant homeostasis (18) and protection from pneumonia

329 (19). Nfkbiz knockout mice are severely deficient in GMCSF (15). Recently, we demonstrated

$330 \mathrm{I} \mathrm{I} \mathrm{B} \zeta$ 's role in regulating inflammatory responses to pneumococcus infection (20), further

331 emphasizing the importance of $\mathrm{I} \kappa \mathrm{B} \zeta$ in lung immune defense. Original studies reported that

332 mononuclear phagocytes which exist in the pristine vascular environment require additional TLR

333 or IL-1R agonists to induce I $\mathrm{I} B \zeta$ expression (8). However, constitutive expression of $\mathrm{I} \kappa \mathrm{B} \zeta$

334 messenger RNA levels in epithelial cells lining various mucosal surfaces, including the eyes and

335 the skin have been reported $(13,14)$. These findings suggest the likelihood that local recognition

336 of PAMPs in exposed tissues can induce a steady state expression of $\mathrm{I} \kappa \mathrm{B} \zeta$. However, here for the

337 first time, we provide strong evidence for constitutive expression of $\mathrm{I} \kappa \mathrm{B} \zeta$ protein in airway

338 epithelium, in the absence of exogenous agonist activity. This unique expression of $\mathrm{I} \kappa \mathrm{B} \zeta$ has

339 been consistently observed in all of our previous studies in which we used immunoblotting to

340 study $\mathrm{I} \kappa \mathrm{B} \zeta$ expression in primary respiratory epithelial cells cultured in the absence of known

341 PAMPs and IL-1 (7, 20). 
K Sundaram et al.

Why recombinant $\mathrm{I} \kappa \mathrm{B} \zeta-\mathrm{S}$ blocked all the nonspecific bands, in addition to the $\mathrm{I} \kappa \mathrm{B} \zeta-\mathrm{L}$

343 band in figure 3B is unclear. However, it is noteworthy that it is the same purified $\operatorname{rI} \kappa \mathrm{B} \zeta-\mathrm{S}$ which

344 was the antigen against which the anti-I $\kappa \mathrm{B} \zeta$ antiserum was generated in our laboratory. It is

345 hence plausible that the non-specific protein fragments that may have sequence similarities with

346 the recombinant protein also get detected by the polyclonal antibody population in the antiserum

347 and as a result, get blocked by the recombinant protein.

348 The other protein detected by the anti-IкB $\zeta$ antiserum, lamin B1, predominantly localizes

349 in the nucleus, where functional $\mathrm{I} \kappa \mathrm{B} \zeta$ also accumulates. This made the interpretation of our

350 immunostaining data difficult. Testing our anti-I $\kappa \mathrm{B} \zeta$ antiserum on airway tissue from mice with

351 conditional complete Nfkbiz knockout in the lungs will be needed to absolutely confirm

352 specificity. Our data highlights the need for a highly sensitive and specific IкB $\zeta$ antibody,

353 especially for use in IHC-based detection of the protein in tissue samples. This study also

354 emphasizes the importance of having the right controls to prove antibody specificity while

355 demonstrating $\mathrm{I} \kappa \mathrm{B} \zeta$ expression in tissues using IHC.

356 Our observation concurs with the known constitutive expression of I $\mathrm{B} \zeta$ in other mucosal

357 surfaces such as skin and eyes, further supporting our findings. I $\kappa \mathrm{B} \zeta$ 's constitutive expression in

358 airway epithelium suggests a function and regulation that may differ from its classical TLR/IL-1

359 induction seen in immune cells. Interestingly, the baseline $\mathrm{I} \kappa \mathrm{B} \zeta$ expression had no apparent

360 function in terms of activating cytokine release from primary human bronchial epithelial cells

361 (7). It is conceivable that $\mathrm{I} \kappa \mathrm{B} \zeta$, like other members of the I $\mathrm{B}$ family, requires post-translational

362 modification such as phosphorylation, as an essential step for its functional activation. Smearing

363 of the $\mathrm{I} \kappa \mathrm{B} \zeta-\mathrm{L}$ band (figure 5A) and its pattern with a slight increase in size of isoforms in the

364 acidic range of the 2D gel (figure 4A) are suggestive of the same. Nevertheless, IL-1 was able to 
K Sundaram et al.

365 induce further $\mathrm{I} \kappa \mathrm{B} \zeta$ protein beyond the baseline expression which was associated with

366 subsequent cytokine release in these cells (7). Future studies evaluating $\mathrm{I} \kappa \mathrm{B} \zeta$ 's signal specific

367 interactions with its known partners like $\mathrm{NF \kappa B}$ or akirin2 (21) or its post-translational

368 modification as an activation step, may be able to identify the exact function of the uniquely

369 expressed $\mathrm{I} \kappa \mathrm{B} \zeta$ in lung epithelium. Nevertheless, our current data support a vital role for I $\mathrm{I} \mathrm{B} \zeta$ in

370 maintaining homeostasis of lung immunity.

\section{CONCLUSION}

372 In conclusion, our data demonstrate constitutive $\mathrm{I} \kappa \mathrm{B} \zeta$ protein expression in the airway

373 epithelium of both humans and mice. This constitutive expression likely is critical to lung host

374 defense via effects on downstream transcriptional targets. Understanding how this constitutive

375 expression of $\mathrm{I} \kappa \mathrm{B} \zeta$ changes during disease conditions will help evaluate the protein as a potential

376 biomarker and a therapy candidate. This study also highlights the need for a highly sensitive and

377 specific antibody for $\mathrm{I} \kappa \mathrm{B} \zeta$ detection, especially for use in immunohistochemistry. 
K Sundaram et al.

\section{DECLARATIONS}

\section{Ethics approval and consent to participate}

394 All animal experiments were performed according to animal protocols approved by the Animal

395 Care Use Committee of the Ohio State University College of Medicine. Anonymized human

396 lung tissue slides were provided by the Ohio State University Department of Pathology, Tissue

397 Archive Service. Other investigators may have received specimens from the same subjects.

\section{Availability of data and materials}

399 All data generated or analyzed during this study are included in this published article [and its

400 supplementary information files]

\section{$401 \quad$ Competing interests}

402 The authors declare that they have no competing interests.

\section{$403 \quad$ Funding}

404 This work was supported by NIH grants HL089440 and HL076278 to Dr. Wewers.

\section{Author Contributions}

$406 \mathrm{KS}^{1}$ and MDW conceived and designed experiments;

$407 \mathrm{KS}^{1}$ and MDW analyzed and interpreted data;

$408 \mathrm{KS}^{1}$ and MDW drafted the manuscript for important intellectual content;

$409 \mathrm{KS}^{1}$ and SM performed experiments;

$410 \mathrm{KSO}$ and SL provided lung tissue from $\mathrm{Nfkbiz}^{-/}$knockout mice;

411 PNB and HS provided lung tissue from gnotobiotic mice; 
K Sundaram et al.

412 AS provided lung tissue from normal wild type mice;

$413 \mathrm{KS}^{6}$ provided human lung tissue slides.

414 All authors reviewed the final manuscript.

415

\section{Acknowledgements}

417 We thank Masami Morimatsu (Sapporo, Japan) for kindly providing us the $\mathrm{Nfkbiz}^{-/-}$knockout 418 mice. We thank Arun Tiwari, a former member of OSU's proteomics facility, for his help with 419 generating the mass spectrometry data. We thank Florinda Jaynes and Alan Flechtner from the 420 Comparative Pathology and Mouse Phenotyping Shared Resource at OSU, for performing IHC

421 on our samples. We thank Sudarshan Seshadri and Yashaswini Kannan for establishing the tools 422 to study $\mathrm{I} \kappa \mathrm{B} \zeta$ in our laboratory. 
K Sundaram et al.

\section{REFERENCES}

440 1. Kitamura H, Kanehira K, Okita K, Morimatsu M, Saito M. MAIL, a novel nuclear I kappa B protein that potentiates LPS-induced IL-6 production. FEBS Lett. 2000;485(1):53-6.

442 2. Haruta $H$. Isolation of a novel interleukin-1-inducible nuclear protein bearing ankyrinrepeat motifs. Journal of Biological Chemistry. 2001;276(16):12485-8. proinflammatory stimuli, negatively regulates nuclear factor-kappaB in the nuclei. J Biol Chem. 2001;276(29):27657-62.

4. Cowland JB, Muta T, Borregaard N. IL-1beta-specific up-regulation of neutrophil gelatinase-associated lipocalin is controlled by IkappaB-zeta. J Immunol. 2006;176(9):5559-66. 5. Kao CY, Kim C, Huang F, Wu R. Requirements for two proximal NF-kappaB binding sites and IkappaB-zeta in IL-17A-induced human beta-defensin 2 expression by conducting airway epithelium. J Biol Chem. 2008;283(22):15309-18.

452 6. Kannan Y, Yu J, Raices RM, Seshadri S, Wei M, Caligiuri MA, et al. IkappaBzeta augments IL-12- and IL-18-mediated IFN-gamma production in human NK cells. Blood. 2011;117(10):2855-63.

7. Sundaram K, Mitra S, Gavrilin MA, Wewers MD. House Dust Mite Allergens and the Induction of Monocyte Interleukin 1beta Production That Triggers an IkappaBzeta-Dependent Granulocyte Macrophage Colony-Stimulating Factor Release from Human Lung Epithelial Cells. American journal of respiratory cell and molecular biology. 2015;53(3):400-11. 8. Seshadri S, Kannan Y, Mitra S, Parker-Barnes J, Wewers MD. MAIL regulates human monocyte IL-6 production. J Immunol. 2009;183(8):5358-68. 9. Hildebrand DG, Alexander E, Horber S, Lehle S, Obermayer K, Munck NA, et al. IkappaBzeta is a transcriptional key regulator of CCL2/MCP-1. J Immunol. 2013;190(9):481220.

10. Kayama H, Ramirez-Carrozzi VR, Yamamoto M, Mizutani T, Kuwata H, Iba H, et al. Class-specific regulation of pro-inflammatory genes by MyD88 pathways and IkappaBzeta. J Biol Chem. 2008;283(18):12468-77.

11. Kitamura H, Matsushita Y, Iwanaga T, Mori K, Kanehira K, Fujikura D, et al. Bacterial lipopolysaccharide-induced expression of the IkappaB protein MAIL in B-lymphocytes and macrophages. Arch Histol Cytol. 2003;66(1):53-62.

470 12. Oonuma T, Morimatsu M, Ochiai K, Iwanaga T, Hashizume K. Role of NF-kappaB in constitutive expression of MAIL in epidermal keratinocytes. J Vet Med Sci. 2007;69(3):279-84. 13. Shiina T, Konno A, Oonuma T, Kitamura H, Imaoka K, Takeda N, et al. Targeted disruption of MAIL, a nuclear IkappaB protein, leads to severe atopic dermatitis-like disease. J Biol Chem. 2004;279(53):55493-8. 
K Sundaram et al.

14. Ueta M, Hamuro J, Yamamoto M, Kaseda K, Akira S, Kinoshita S. Spontaneous ocular surface inflammation and goblet cell disappearance in I kappa B zeta gene-disrupted mice. Invest Ophthalmol Vis Sci. 2005;46(2):579-88.

478 15. Yamamoto M, Yamazaki S, Uematsu S, Sato S, Hemmi H, Hoshino K, et al. Regulation of Toll/IL-1-receptor-mediated gene expression by the inducible nuclear protein IkappaBzeta. Nature. 2004;430(6996):218-22.

481 16. Lorenz J, Zahlten J, Pollok I, Lippmann J, Scharf S, N'Guessan PD, et al. Legionella 482 pneumophila-induced IkappaBzeta-dependent expression of interleukin-6 in lung epithelium. Eur 483 Respir J. 2011;37(3):648-57.

484 17. Seshadri S. Role of IkappaBzeta and Pyrin as Modulators of Macrophage Innate Immune 485 Function: The Ohio State University; 2008.

486 18. Huffman JA, Hull WM, Dranoff G, Mulligan RC, Whitsett JA. Pulmonary epithelial cell 487 expression of GM-CSF corrects the alveolar proteinosis in GM-CSF-deficient mice. JClinInvest. 488 1996;97(3):649-55.

489 19. Trapnell BC, Whitsett JA. Gm-CSF regulates pulmonary surfactant homeostasis and alveolar macrophage-mediated innate host defense. AnnuRev Physiol. 2002;64:775-802. 20. Sundaram K, Rahman MA, Mitra S, Knoell DL, Woodiga SA, King SJ, et al. IkappaBzeta Regulates Human Monocyte Pro-Inflammatory Responses Induced by Streptococcus pneumoniae. PloS one. 2016;11(9):e0161931. critical for inducing inflammatory genes by bridging IkappaB-zeta and the SWI/SNF complex. EMBO J. 2014;33(20):2332-48. 
K Sundaram et al.

\section{FIGURE LEGENDS}

515 Figure 1. IкB $\zeta$ expression in normal lung tissue. Lung tissue slices from (A) healthy human

516 individuals were immunostained with secondary antibody $\left(2^{\circ}\right.$ ab) alone $(1: 1000)$, rabbit control

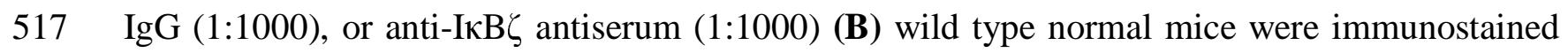

518 with secondary antibody alone (1:1000), IgG isotype control (1:2000), or anti-I $\mathrm{B} \zeta$ antiserum

519 (1:2000), observed at 100, 400 and 1000X magnifications. The scale bar denotes $50 \mu \mathrm{m}$ for the

$5201000 \mathrm{X}$ magnification panels. The results are representative of 3 different mice/humans. Red

521 boxes represent regions that were captured at higher magnifications.

522 Figure 2. IкB $\zeta$ expression in lung tissue from gnotobiotic and $\mathrm{Nfkbiz}^{-/}$knockout mice. Lung

523 tissue slices from (A) gnotobiotic mice were immunostained with secondary antibody $\left(2^{\circ}\right.$ ab)

524 alone (1:1000), IgG isotype control (1:1500), or anti-IкB $\zeta$ antiserum (1:1500), observed at 100,

525400 and 1000X magnifications and from (B) $\mathrm{Nfkbiz}^{-/-}$knockout mice were immunostained with

526 IgG isotype control (1:2500), or anti-I $\kappa \mathrm{B} \zeta$ antiserum (1:2500), observed at 400X magnification.

527 The scale bar denotes $50 \mu \mathrm{m}$ for the $1000 \mathrm{X}$ magnification panels. The results are representative

528 of 3 different mice. Red boxes represent regions that were captured at higher magnifications.

529 Figure 3. rIкB $\zeta$ blocks non-specific signal detected by anti-IкB $\zeta$ antiserum (A) Immunoblot

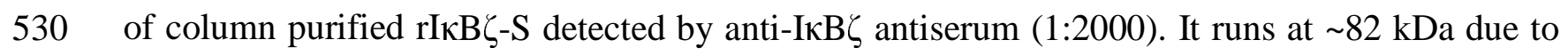

531 the presence of His and thioredoxin tags. (B) IL-1 $\beta$-stimulated BEAS2B cell extracts were 
K Sundaram et al.

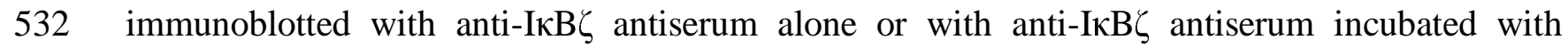

533 excess $\operatorname{rI} \kappa \mathrm{B} \zeta-\mathrm{S}$ protein. Asterisk represents non-specific protein detected. Beta actin was used as

534 protein loading control. The results are representative of 3 independent experiments.

535 Figure 4. Anti-IкB $\zeta$ antiserum detects lamin B1 protein. (A) Lysates of HeLa cells stimulated

536 with IL-1 $\beta$ were immunoprecipitated using anti-IкB $\zeta$ antiserum (right panel) or IgG control (left

537 panel) and run on 2D SDS-PAGE, followed by immunoblotting with anti-IkB $\zeta$ antiserum

538 (1:2000). White arrow head represents lamin B1 while black arrow represents $\mathrm{I} \kappa \mathrm{B} \zeta-\mathrm{L}$, as

539 identified by ESI-TRAP mass spectrometry. White arrow represents IgG heavy chain. (B)

540 Lysates of monocytes stimulated with LPS were immunoprecipitated using anti-lamin B1

541 antibody and blotted with respective antibodies as indicated. The results are representative of 3

542 independent experiments.

543 Figure 5. Immunoblotting demonstrates constitutive IкB $\zeta$ expression in airway epithelium.

544 (A) Immunoblot of homogenates from trachea and lungs of gnotobiotic mice, detected by anti-

$545 \mathrm{I} \kappa \mathrm{B} \zeta$ antiserum. The results are representative of 3 different mice. (B) HBECs $\left(2 \times 10^{5}\right.$ cells per

$546 \mathrm{ml})$ were stimulated with rhIL-1 $\beta(10 \mathrm{ng} / \mathrm{ml})$ for 3 hours, with or without the pretreatment with

547 IL-1Ra $(10 \mu \mathrm{g} / \mathrm{ml})$. Cell extracts were immunoblotted for $\mathrm{I} \kappa \mathrm{B} \zeta$ with anti-I $\mathrm{B} \zeta$ antiserum

548 (1:2000). IкB $\zeta$ expression was normalized to protein loading control and represented as

549 densitometric units. Error bars represent the mean \pm SEM of 3 independent experiments. NT

550 stands for no treatment control. Beta actin was used as protein loading control for both (A) and

551 (B).

552 Supplementary Figure S1. Anti-IкB $\zeta$ antiserum detects IкB $\zeta$ protein. (A) BEAS2B cells

553 were $\left(1.5 \times 10^{5}\right.$ cells $\left./ \mathrm{ml}\right)$ transfected with $100 \mathrm{pmol}$ of scrambled siRNA control or siRNA specific

554 to I $\kappa \mathrm{B} \zeta$ and stimulated with rhIL-1 $\beta(10 \mathrm{ng} / \mathrm{ml})$ for 3 hours. Cell lysates were immunoblotted for 
K Sundaram et al.

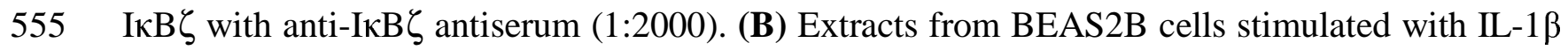

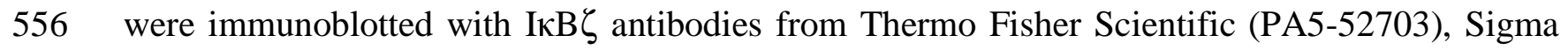

557 Aldrich (HPA010547), Abcam (ab221914) and LS Biosciences (LS-C294627) at their

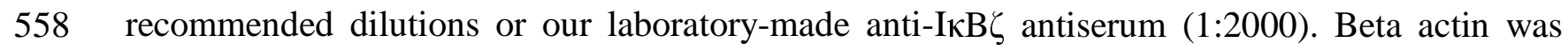

559 used as equal protein loading control for both (A) and (B). Asterisk represents non-specific

560 proteins detected. The results are representative of at least 3 independent experiments.

561 Supplementary Figure S2. IkB $\zeta$ expression in trachea from gnotobiotic mice. Tracheal slices

562 from gnotobiotic mice were immunostained with secondary antibody alone $\left(2^{\circ} \mathrm{ab}\right)(1: 1000), \operatorname{IgG}$

563 isotype control (1:1500), or anti-IкB $\zeta$ antiserum (1:1500), observed at 100, 400 and $1000 \mathrm{X}$

564 magnifications. The scale bar denotes $50 \mu \mathrm{m}$ for the $1000 \mathrm{X}$ magnification panels. The results are

565 representative of 3 different mice. Red boxes represent regions that were captured at higher

566 magnifications.

567 Supplementary Figure S3. THP-1 nuclear extract blocks non-specific IgGs in anti-IкB $\zeta$

568 antiserum. (A) Immunoblot of non-specific lamin B1 protein from the nuclear extract (NE),

569 cytoplasmic extract $(\mathrm{CE})$ and nuclear pellet $(\mathrm{Nu})$ of unstimulated THP-1 cells, using Lamin B1

570 antibody at its recommended dilution. IL-1 $\beta$ stimulated BEAS2B cell extracts (B)

571 immunoblotted with our laboratory made anti-IкB $\zeta$ antiserum alone (1:5000) or with the anti-

$572 \mathrm{I} \kappa \mathrm{B} \zeta$ antiserum pre-cleared with excess unstimulated THP-1 NE and (C) immunostained with

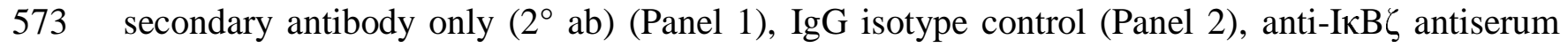

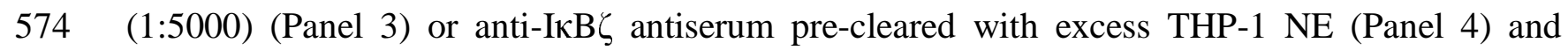

575 imaged at 100X magnification. NT stands for no treatment. The results are representative of 7-8

576 independent experiments. 
bioRxiv preprint doi: https://doi.org/10.1101/255331; this version posted March 29, 2019. The copyright holder for this preprint (which was not certified by peer review) is the author/funder. All rights reserved. No reuse allowed without permission.

K Sundaram et al. 
A.

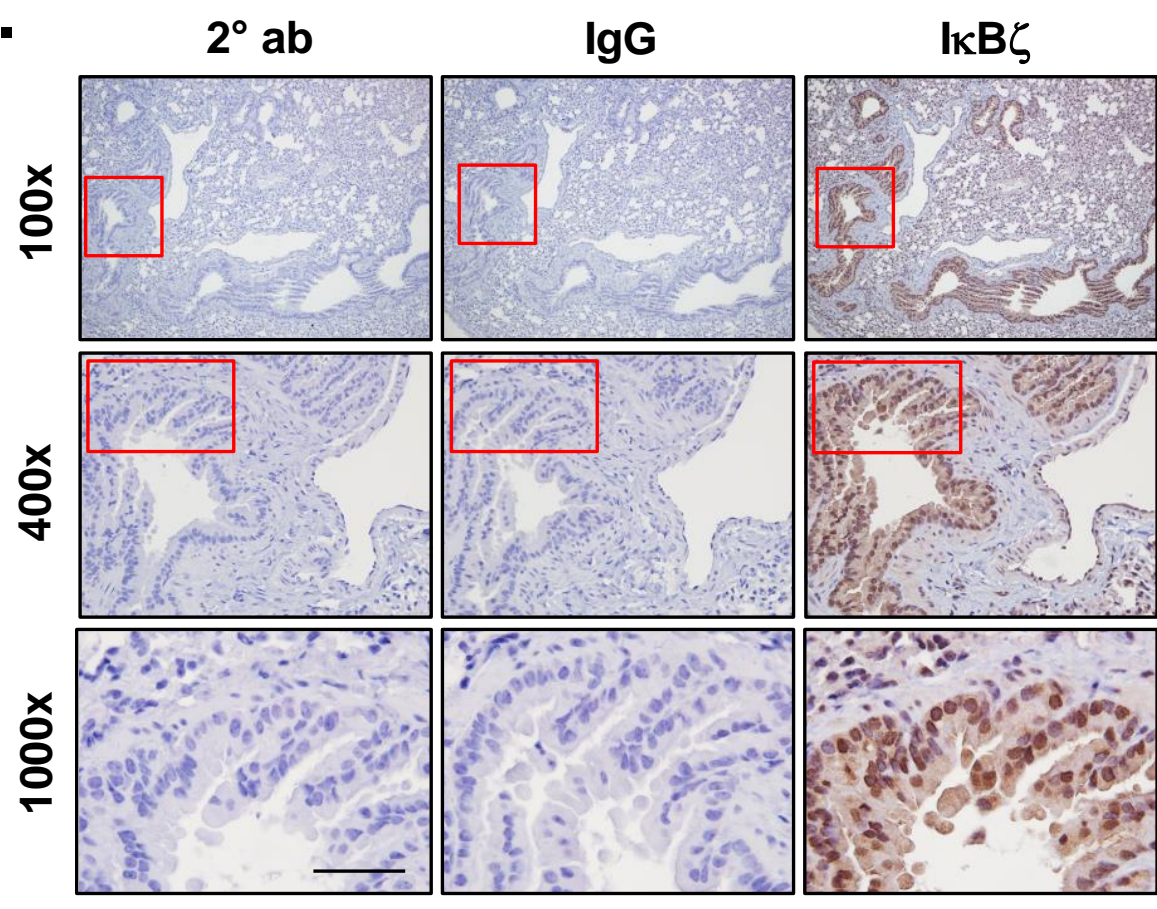

B.

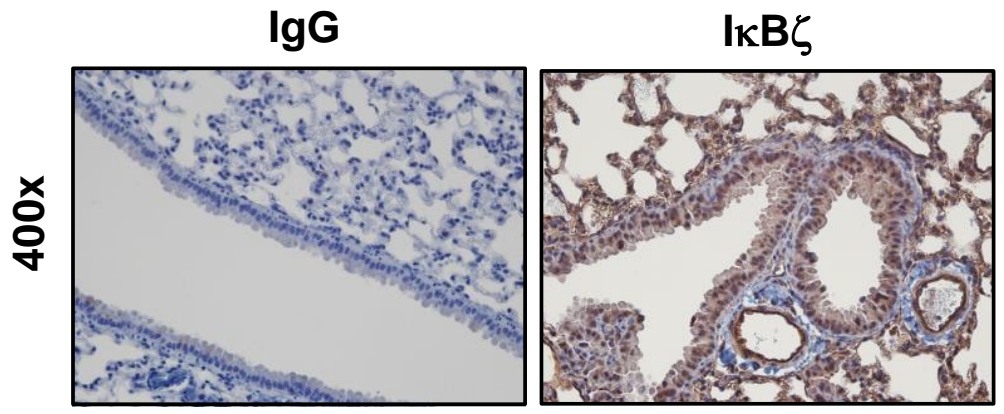

Revised

Fig 2 
A.

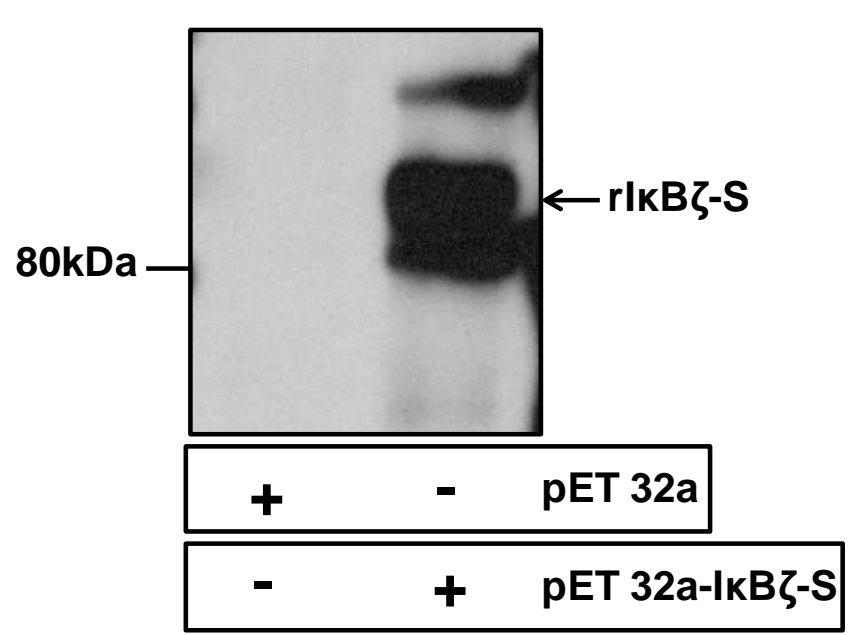

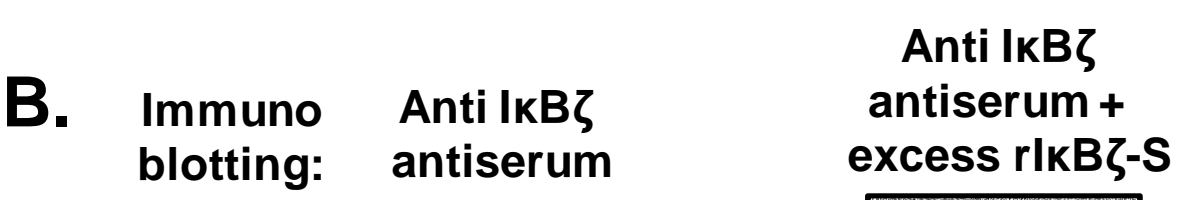

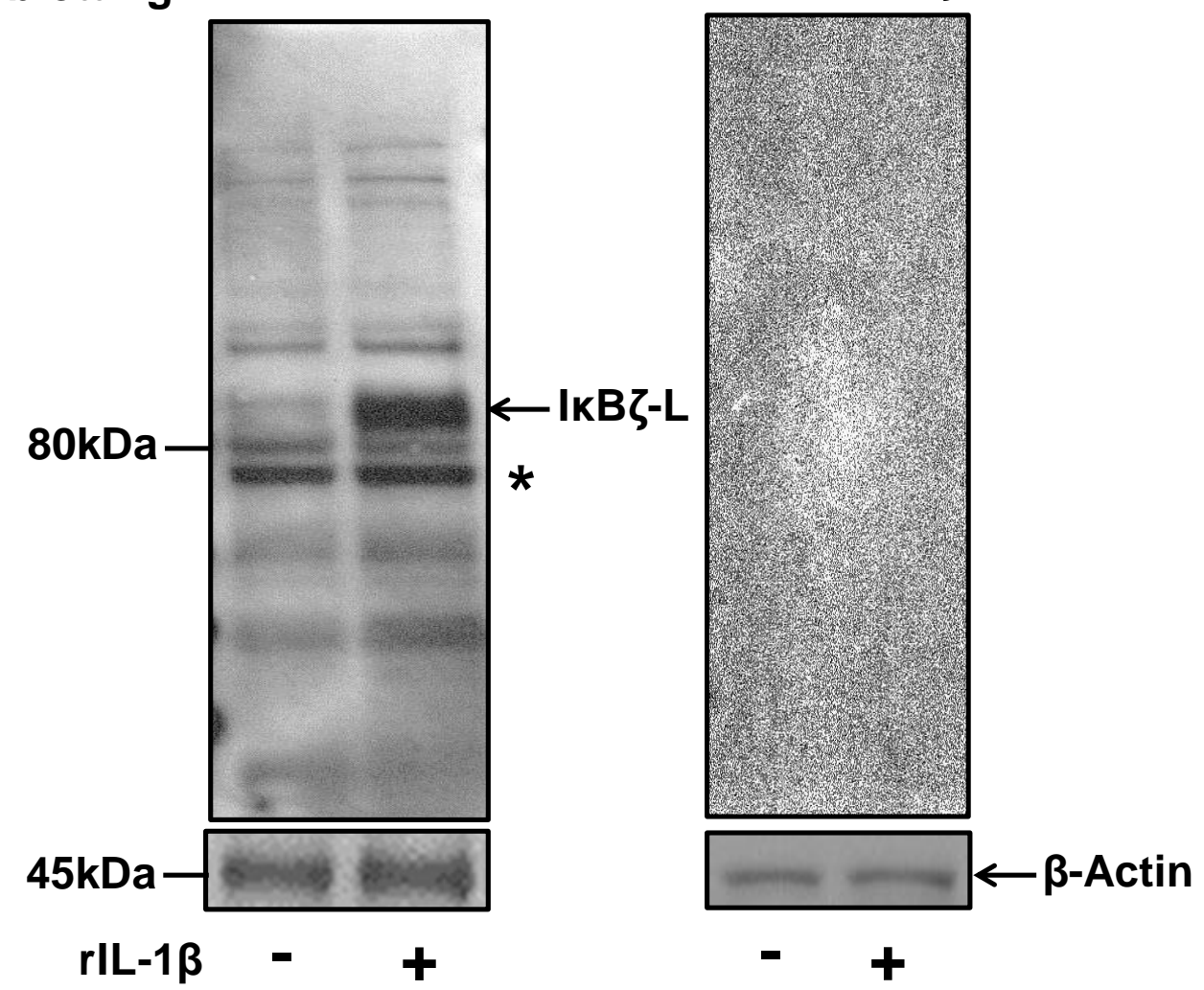

Revised

Fig 3 
A.
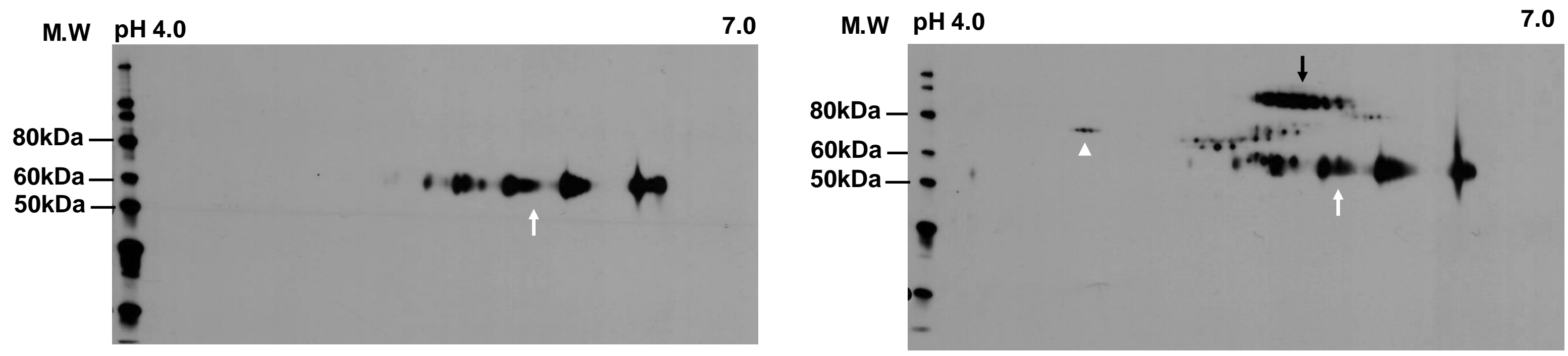

B.

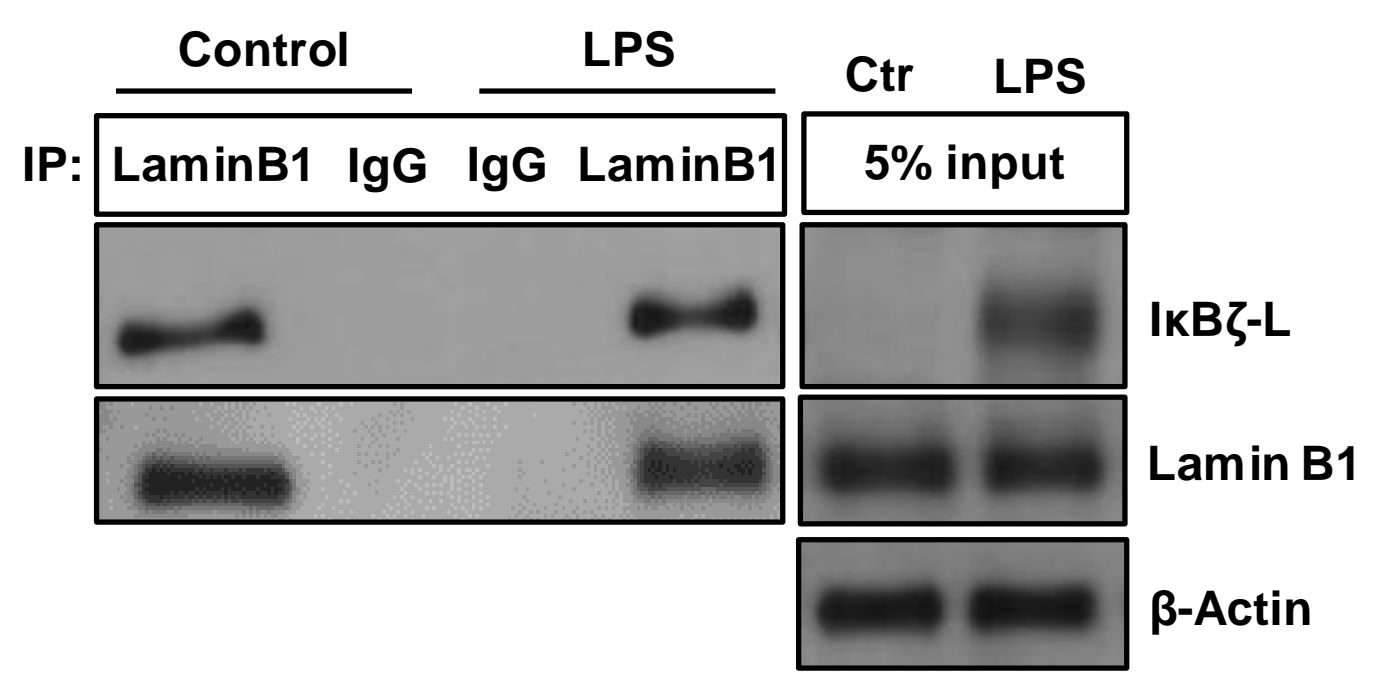

Revised 
A.

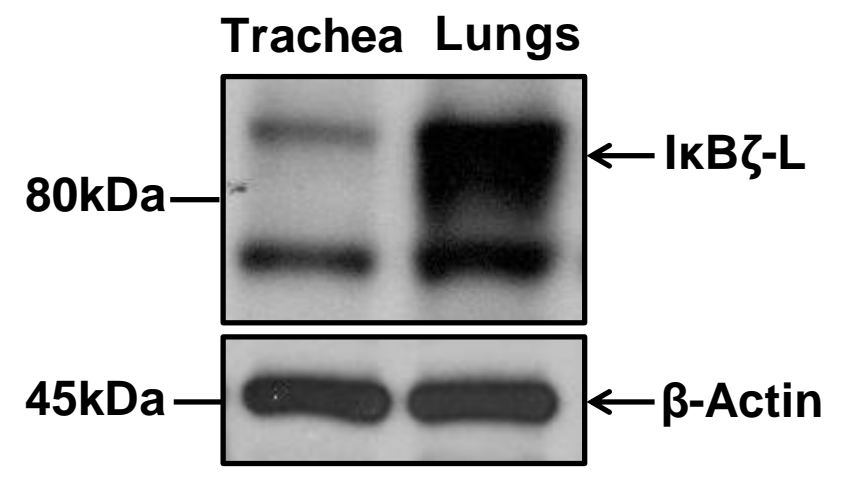

B.

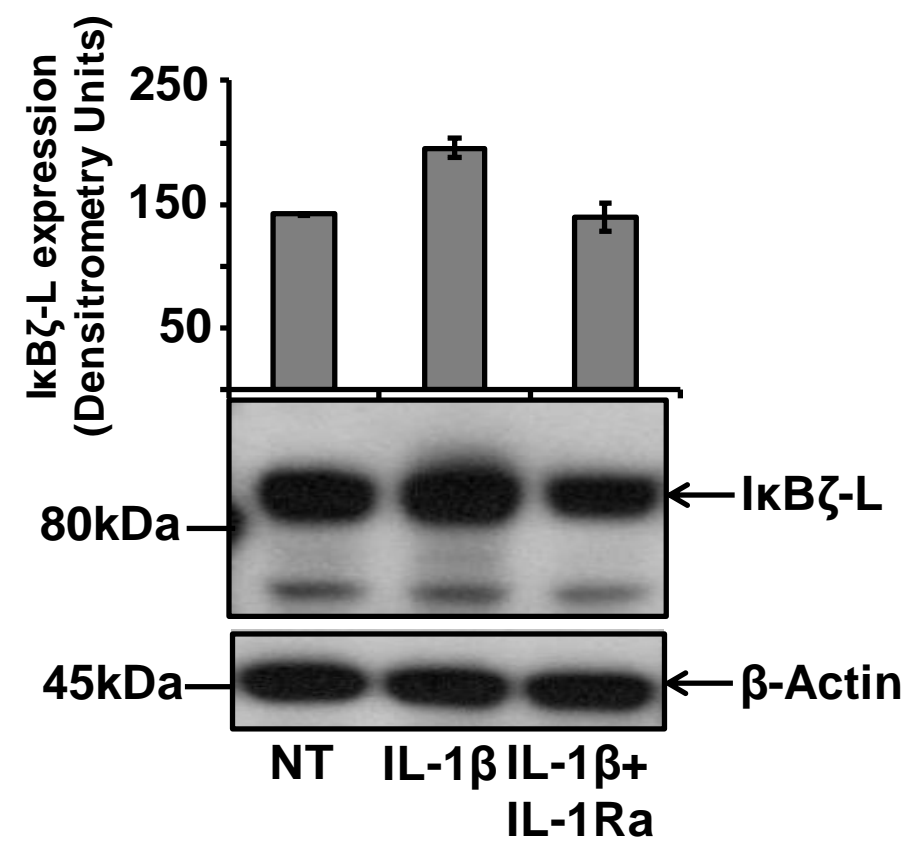

Revised

Fig 5 